\section{Assurance et santé: comment ça marche?}

\author{
T. Perneger, ${ }^{a, b}$ P. Bovier, ${ }^{b, d}$ P. Chopard, ${ }^{b, f}$ P. Garnerin, ${ }^{b, g}$ \\ E. Chamot, ${ }^{a}$ F. Herrmann, ${ }^{b, e}$ P. Chastonay, ${ }^{a, c}$
}

a Institut de médecine sociale et préventive, Université de Genève

b Unité qualité des soins, Hôpitaux Universitaires de Genève

c Unité de développement et de recherche en éducation médicale

d Département de médecine communautaire,

Hôpitaux Universitaires de Genève

e Hôpital de Gériartrie, HUG

f Département de médecine interne, HUG

g Division d'anesthésiologie, HUG

\begin{abstract}
Résumé
Cet article propose une revue des diverses fonctions de l'assurance dans le domaine de la santé et des soins, de ses effets désirables et indésirables, des mesures envisageables visant à limiter l'impact de ces derniers, et des différences entre assurance commerciale et assurance sociale.
\end{abstract}

\section{Introduction}

Actuellement, en Suisse, on entend souvent dire que l'assurance-maladie va mal. Avant d'accepter ou de réfuter cette opinion, et pour orienter les éventuelles réformes de cette institution, il convient de comprendre à quoi sert une assurance dans le domaine de la santé, quelles sont ses retombées positives mais aussi négatives, et de quels outils on dispose pour limiter les éventuels effets pervers.

\section{Le principe de l'assurance}

Un marché de l'assurance apparaît chaque fois que la population est exposée à un risque imprévisible d'un événement entraînant de graves répercussions financières, tel que décès, incendie, ou maladie grave. L'événement doit être imprévisible, sans quoi les frais générés par l'assurance elle même ne se justifieraient pas, et ses conséquences doivent être financières.

Correspondance:

Dr Philippe Chastonay

Centre médical universitaire

Institut de médecine sociale et préventive

Rue Michel-Servet

CH-1211 Genève 4
Ce qui permet à une assurance de justifier ses frais est qu'en général, les gens attribuent d'autant moins de valeur à une somme d'argent qu'ils en ont davantage (songeons à ce que représentent cent francs pour un mendiant ou pour Bill Gates). En jargon économique, ceci s'appelle une «utilité marginale décroissante». Par symétrie, on perd moins d'utilité à payer régulièrement une prime d'assurance raisonnable qu'à verser d'un seul coup une somme qui nous mettrait en faillite. Ainsi l'assurance génère, comme par magie, du bien-être social, en répartissant entre payeurs multiples les conséquences financières des événements indésirables et rares.

La plupart des gens seront même prêts à payer plus que ce qui serait strictement justifié par le risque encouru. Si 1000 villas à 1 million de francs chacune sont assurées contre l'incendie, et qu'il survient en moyenne un incendie par année, la prime d'assurance-incendie correcte serait de 1000 francs par villa par an. Or, grâce à l'utilité marginale décroissante de l'argent, l'assureur peut se permettre de demander plus que 1000 francs, disons 1200, ce qui lui permet de couvrir ses frais et de faire des bénéfices. On peut aussi se représenter la plus-value que produit une assurance de façon plus intuitive: la plupart des gens n'aiment guère le risque (ici, risque financier causé par la maladie) et sont prêts à payer un peu trop pour éviter cette épée de Damoclès; l'assurance fournit précisément ce service.

\section{Meilleur accès aux soins et meilleure santé}

Dans le cas de l'assurance-maladie, d'autres objectifs utiles peuvent être atteints, notamment celui d'une équité dans l'accès aux soins. Si la consommation des soins était limitée par le pouvoir d'achat, comme il se doit dans toute économie de marché, l'état de santé d'une partie de la population risquerait de se détériorer. Ceci est politiquement inacceptable dans la plupart des pays développés, où l'accès aux soins est considéré comme un droit fondamental de l'individu. Le risque pour la santé encouru par les personnes démunies n'est pas seulement théorique. Dans les années 1980, l'état de Californie a aboli son assurancemaladie pour indigents, et on a pu constater alors une détérioration rapide et sérieuse de l'état de santé des patients diabétiques et hypertendus ainsi livrés à eux mêmes [1].

L'objectif de l'équité d'accès explique pourquoi l'assurance-maladie s'étend habituellement à toutes les dépenses de santé jugées utiles, et pas seulement aux frais catastrophiques, comme suggéré dans le paragraphe précédent. Grâce à cet élargissement des événements couverts, l'assurance-maladie contribue au maintien de l'état de santé de la population. En plus, en faisant jouer la solidarité entre les sous groupes favorisés et défavorisés, par le jeu des primes et subsides, l'assurance-maladie "sociale» augmente la cohésion sociale. 
Tableau 1

Maîtrise des coûts de la santé lorsqu'une assurance-maladie est en place.

\begin{tabular}{lll}
\hline Type de mesure & Exemples & Utilisé en Suisse \\
\hline Mesures générales & Liste limitative des prestations couvertes & Oui \\
& Tarifs contrôlés & Oui \\
\hline Diminution de l'offre & Liste limitative de médecins et hôpitaux & Oui (HMO) \\
& Approbation du médecin-conseil nécessaire pour certains soins & Oui (HMO) \\
& Paiement des soignants indépendant des prestations & Oui (HM0) \\
\hline Diminution de la demande & Participation des assurés aux frais & Oui \\
& Franchise & Oui \\
\hline
\end{tabular}

\section{L'assurance fait grimper les coûts de la santé}

Malheureusement, l'assurance modifie le comportement des acteurs en présence. Les assurés prendront généralement moins de précautions pour éviter l'événement indésirable, tel le conducteur qui, ayant contracté une assurance casco, fera moins attention à ne pas rayer sa carrosserie. Ceci constitue un changement de comportement induit par l'assurance ("moral hazard" dans le jargon économique, "ex ante» parce que ce changement précède la survenue de l'événement assuré). Dans le domaine de la santé, cependant, on n'a jamais démontré que l'assurancemaladie diminue les comportements de prévention.

L'assuré peut aussi changer de comportement après l'événement: un assuré qui a subi un dommage voudra une réparation parfaite, alors que sans assurance il se contenterait d'une réparation minimale. C'est cette forme de changement de comportement induit ("moral hazard ex-post») qui est la plus importante dans le secteur de la santé: l'assuré qui éprouve un symptôme consultera plus rapidement, et exigera des investigations et des soins plus poussés qu'une personne devant payer chaque acte de sa poche. Donc les coûts de la santé augmenteront. Attention: l'augmentation des recours aux soins est en partie souhaitable, notamment pour les personnes défavorisées, mais compte tenu de l'impossibilité dans laquelle se trouve le patient (et bien souvent le médecin) de distinguer entre soins utiles et superflus, on suspecte que l'augmentation de la consommation des soins est en partie injustifiée.

Les médecins et hôpitaux subissent une pression semblable. Lorsque les prix des prestations n'influencent pas les décisions de consulter, il n'y a pas de concurrence possible sur les prix, fondement de l'efficience des marchés selon la théorie économique classique. Dans cette situation, la seule façon pour un fournisseur de soins de prendre une part de marché est d'offrir des services différents, meilleurs, plus évolués technologiquement (par exemple, proposer un plâtre en époxy là où son analogue en vrai plâtre ferait l'affaire). Habituellement, de tels services coûtent cher, et les dépenses de santé augmentent en conséquence.
Autre conséquence de l'absence de concurrence sur les prix: les prestataires de soins n'ont pas d'intérêt évident à faire marcher la concurrence entre leurs fournisseurs (par exemple, trouver un fournisseur de plâtre plus avantageux). Cela aboutit parfois à des prix absurdement élevés des fournitures médicales. Par ces différents mécanismes, la généralisation d'une assurance-maladie fait nécessairement augmenter les coûts des soins.

\section{Maîtrise des coûts}

La maîtrise des coûts de la santé est donc une préoccupation primordiale dès qu'un système d'assurance est en place. Ce thème est discuté en détail dans un autre article; ici, nous abordons uniquement les moyens directement liés au mécanisme d'assurance (tab. 1).

D’abord, il existe une série de mesures générales: on peut limiter le catalogue des prestations couvertes par l'assurance, et imposer un tarif contraignant. En Suisse, la Loi sur l'assurance-maladie (LAMal) applique ces mesures [2]. On peut aussi limiter l'offre, soit en restreignant la liste des fournisseurs de soins admissibles, soit en exigeant une approbation par le médecin-conseil de l'assurance pour les soins chers; ceci est typiquement fait dans les caisses-maladie de type "managed care" ou "health maintenance organization" (caisses-maladie avec choix limité du médecin selon la LAMal). Dans ces organisations, on applique également un mode de rétribution des soignants qui favorise les pratiques économiques (voir article suivant).

On peut aussi limiter la demande des soins, en demandant à l'assuré d'assumer une partie des frais qu'il génère, pour qu'il s'abstienne de consulter pour des motifs futiles. Ces participations réduisent les coûts, comme cela a été démontré par une étude expérimentale américaine (RAND Health Insurance Experiment) [3]. Malheureusement, ce sont les plus démunis qui sont les plus sensibles à ces effets. Le rôle de la franchise annuelle est apparenté. La franchise est censée aussi limiter les frais administratifs des caisses-maladie causés par le traitement des factures, même de faible montant. 
Tableau 2

Modes de financement des principales assurances santé en Suisse.

\begin{tabular}{lll}
\hline Assurance & Financement & Redistribution \\
\hline Complémentaire & Privé, en fonction du risque & Aucune \\
\hline Assurance-maladie de base & $\begin{array}{l}\text { Primes fixes pour chaque caisse dans un canton } \\
\text { donné; subventions pour revenus bas }\end{array}$ & $\begin{array}{l}\text { Entre bien-portants et malades, jeunes et vieux; } \\
\text { partielle entre classes de revenu } \\
\text { (via les subventions), aucune entre cantons }\end{array}$ \\
\hline Assurance-accidents & $\begin{array}{l}\text { Primes en pour-cent du revenu (plafonné), } \\
\text { varient par secteur d'activité }\end{array}$ & $\begin{array}{l}\text { Entre revenus bas et hauts, } \\
\text { mais pas entre secteurs d'activité économique }\end{array}$ \\
\hline Assurance-militaire & Impôt & $\begin{array}{l}\text { En fonction du revenu, état de santé, } \\
\text { âge et sexe, etc. }\end{array}$ \\
\hline
\end{tabular}

\section{Assurance sociale}

Lorsqu'on contracte une police d'assurance commerciale, la prime est calculée en fonction du risque individuel de sinistre. Ceci est dicté par les lois du marché. Si la prime n'était pas fonction du risque, ceux à qui on demande de payer plus que nécessaire refuseraient à juste titre d'adhérer, le coût moyen des adhérents augmenterait, des personnes supplémentaires devraient renoncer à l'assurance, et ainsi de suite jusqu'à l'effondrement du système. En Suisse, le principe de la prime proportionnelle au risque s'applique aux assurances maladie complémentaires, où la prime est calculée en fonction de l'état de santé de l'assuré.

En revanche, dans une assurance dite sociale, certains vont payer plus, et d'autres moins, que ne le voudrait leur niveau de risque individuel. La redistribution des charges que cela implique poursuit un but politique au-delà de la couverture du risque financier lié à la maladie: celui de promouvoir l'équité dans le domaine des soins de santé. Ainsi, on peut souhaiter, comme c'est le cas pour la LAMal, que les malades et les bien-portants paient la même prime, même si leur risque moyen de consommer des soins diffère évidemment. Notons que la définition de l'équité peut varier. On peut estimer en effet qu'une prime identique pour tous n'est pas vraiment équitable, mais que la prime doit être adaptée à la capacité de payer, à savoir au revenu (voir plus bas).

Une conséquence de ces mécanismes de redistribution est que l'assurance sociale doit être universelle, d'une part pour que chacun participe à l'effort de solidarité, mais aussi pour que chacun bénéficie des prestations. Ceci est acquis si l'assurance est financée par l'impôt. Si les primes sont payées par les individus, l'affiliation à l'assurance-maladie doit être obligatoire. Sans cela, comme on l'a vu précédemment, ceux qui s'estiment être désavantagés par le niveau des primes se désengageraient du système, qui finirait par s'effondrer. De plus, l'état devrait lors subventionner les personnes les plus défavorisées, qui pourraient ne pas être à même de payer les primes. Puisqu'une assurance, même facultative, fait monter les prix des soins, les pauvres sans assurance seraient doublement défavorisés: non seulement ils ne pourraient pas payer les primes d'assurance, mais ils de- vraient encore faire face à des prix de services «anormalement» élevés.

Autre corollaire: l'assurance sociale doit imposer un catalogue de prestations couvertes, sans quoi certains pourraient prendre une assurance minimale, évitant de faire l'effort de solidarité. La liste de prestations couvertes par l'assurance de base (LAMal) a donc deux fonctions distinctes et opposées: d'une part, c'est une liste limitative qui cherche à éviter la surconsommation de soins d'efficacité douteuse, d'autre part, c'est une liste minimale de prestations auxquelles chacun a droit. Cette double fonction explique en partie les mésententes et conflits que peut provoquer l'inclusion ou l'exclusion de telle prestation.

\section{Financement de l'assurance}

La redistribution des charges de l'assurance peut prendre plusieurs formes. En Suisse (tab. 2), l'assurance-maladie de base fonctionne avec une prime unique pour tous les adultes d'un canton et d'une caisse-maladie. Ce système impose un transfert des coûts entre bien-portants et malades, hommes et femmes, jeunes et vieux. En revanche, pas de redistribution entre les cantons. La redistribution entre les caisses-maladie est partielle, puisque les caisses dont les assurés sont jeunes alimentent un fonds de compensation destiné aux caisses dont les assurés sont âgés, mais ceci n'efface pas complètement les différences de tarifs entre les caisses. Les primes uniques signifient qu'aucun effort de solidarité n'est demandé aux riches à l'égard des pauvres; seules les subventions fédérales distribuées par les cantons aux personnes à faible revenu vont dans ce sens.

D'autres modes de financement coexistent en Suisse. Les primes d'assurance-accidents diffèrent par secteur d'activité économique, et sont calculées en pour cent du revenu, avec un plafond. Notons que des primes proportionnelles au revenu ont été proposées pour l'assurance-maladie, mais sèchement rejetées en votation populaire en 1994. Un tel financement introduit une redistribution entre personnes à revenu bas et élevé, qui est encore plus marquée lorsque le financement se fait par l'impôt, comme au Royaume 
Uni. En Suisse, l'assurance militaire est financée par l'impôt, mais si tout le monde contribue, seuls les militaires en bénéficient.

\section{Le problème de la sélection}

L'assurance-maladie en Suisse tente d'appliquer deux principes essentiellement contradictoires: la solidarité et la concurrence. La contradiction tient à ceci: la solidarité implique un produit d'assurance identique à prix qui n'est pas conforme aux lois du marché, alors que la concurrence exige une différenciation des produits et une fluctuation libre des prix. Intégrer les deux contraintes tient de la quadrature du cercle. En pratique, une certaine concurrence existe entre caisses-maladie et entre régimes d'assurance (franchise variable, assurance «avec choix limité du médecin" ou HMO). Ceci pose un autre problème: le risque de sélection inégale des assurés.

En effet, si les primes ne reflètent pas le niveau de risque, une caisse qui assure une population en bonne santé fera des bénéfices, alors qu'une caisse assurant une population malade sera en faillite. Une caissemaladie aura donc intérêt à attirer des clients a priori peu coûteux. On peut supposer que c'est pour cela que les affiches des caisses-maladie représentent souvent un couple dans la trentaine avec deux enfants (un couple sans enfant risquerait d'en avoir, ce qui occasionne des frais), ou que certaines caisses remboursent en partie l'abonnement de fitness (une personne chroniquement malade est peu susceptible de prendre un tel abonnement). Le fonds de compensation entre caisses évoqué plus haut n'est que d'une efficacité limitée, puisque l'âge et le sexe (les deux variables prises en compte) n'expliquent qu'environ 5\% de la variance des coûts annuels.

L'autre mesure de la LAMal qui tente de limiter la sélection est le libre passage des assurés d'une caisse à l'autre. Le législateur espère que les assurés appartenant à des caisse-maladie "chères" vont migrer vers celles qui le sont moins, un rééquilibrage se faisant spontanément. Ce scénario exigerait que ce soient surtout les assurés "chers" qui changent de caisse en priorité, or les personnes les plus malades ont avantage à garder la même assurance complémentaire, qui ne bénéficie pas de la clause de libre passage. Si par contre les assurés en bonne santé sont les plus susceptibles de migrer vers les caisses les moins chères, les disparités entre les collectifs des différentes caisses s'en trouveraient accentuées. Le retrait brutal en 1998 d'une grande caisse-maladie suisse du marché de l'assurance de base dans plusieurs cantons non rentables montre les limites de ce mécanisme de régulation.

Les mêmes phénomènes surviennent pour les collectifs assurés avec une franchise à option, ou ceux des HMO. Si leurs assurés consommaient a priori moins de services de santé que ceux des caisses traditionnelles, les coûts de la santé réduits dans ces collectifs ne seraient pas de vraies économies, mais des conséquences d'une distribution inégale des risques. Certaines données suggèrent que ceci s'est produit en Suisse: lorsque l'Université de Genève a introduit en 1992 une assurance HMO pour ses étudiants, ceux qui avaient refusé ce changement ont coûté environ 30\% de plus que ceux qui ont accepté l'HMO au cours de l'année précédant l'introduction du nouveau système [4]. Une solution radicale au problème de la sélection consisterait à créer une caissemaladie unique, à la manière de la Suva pour l'assurance-accidents.

\section{Conclusion}

Ce survol montre que l'assurance-maladie modifie de façon fondamentale le système de santé. Aux côtés des avantages indiscutables (meilleur accès aux soins, meilleure santé, protection contre les effets financiers des maladies), l'assurance induit des coûts de la santé plus élevés, qu'il convient de maîtriser. Le principe de redistribution des charges propre aux assurances sociales entre en contradiction avec le jeu de la concurrence entre les caisses. Le bon fonctionnement de l'assurance-maladie sociale constitue un défi crucial pour le bien-être de la population.

\section{Références}

1 Lurie N, Ward NB, Shapiro MF, Gallego C, Vaghaiwalla R, Brook RH. Termination of Medi-Cal benefits. A follow-up study one year later. N Engl J Med 1986;314:1266-8.

2 Texte de la LAMal: http://www.admin.ch/ch/f/rs/832_10/index.html.

3 Newhouse JP, Manning WG, Morris CN, Orr LL, Duan N, Keeler EB, et al. Some interim results from a controlled trial of cost sharing in health insurance. N Engl J Med 1981; 305:1501-7.

4 Etter JF, Perneger TV. Health care expenditures after introduction of a gatekeeper and a global budget in a Swiss health insurance plan. J Epidemiol Community Health 1998;52:370-6. 